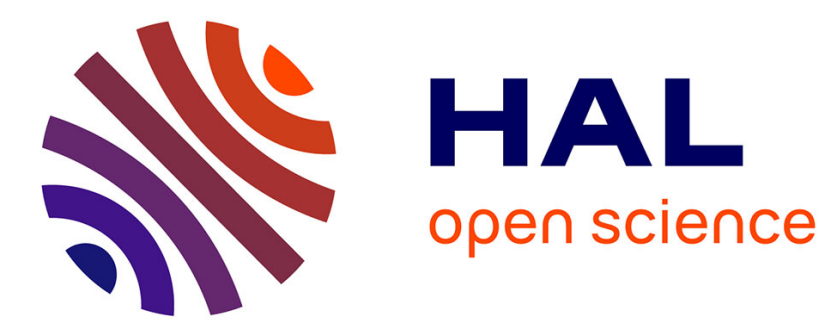

\title{
Time-Varying Parameter Identification Algorithms: Finite and Fixed-Time Convergence
}

Hector Ríos, Denis Efimov, Jaime A Moreno, Wilfrid Perruquetti, J G Rueda-Escobedo

\section{- To cite this version:}

Hector Ríos, Denis Efimov, Jaime A Moreno, Wilfrid Perruquetti, J G Rueda-Escobedo. Time-Varying Parameter Identification Algorithms: Finite and Fixed-Time Convergence. IEEE Transactions on Automatic Control, 2018, 10.1109/TAC.2017.2673413 . hal-01470957

\section{HAL Id: hal-01470957 https://hal.inria.fr/hal-01470957}

Submitted on 18 Feb 2017

HAL is a multi-disciplinary open access archive for the deposit and dissemination of scientific research documents, whether they are published or not. The documents may come from teaching and research institutions in France or abroad, or from public or private research centers.
L'archive ouverte pluridisciplinaire HAL, est destinée au dépôt et à la diffusion de documents scientifiques de niveau recherche, publiés ou non, émanant des établissements d'enseignement et de recherche français ou étrangers, des laboratoires publics ou privés. 


\title{
Time-Varying Parameter Identification Algorithms: Finite and Fixed-Time Convergence ${ }^{\star}$
}

\author{
H. Ríos ${ }^{\dagger}$, D. Efimov ${ }^{\ddagger *}$, J.A. Moreno ${ }^{\S}$, W. Perruquetti ${ }^{\ddagger}$ and J.G. Rueda-Escobedo ${ }^{\S}$
}

\begin{abstract}
In this paper the problem of time-varying parameter identification is studied. To this aim, two identification algorithms are developed in order to identify time-varying parameters in a finite-time or prescribed time (fixed-time). The convergence proofs are based on a notion of finite-time stability over finite intervals of time, i.e. Short-finite-time stability; homogeneity for time-varying systems; and Lyapunov-based approach. The results are obtained under injectivity of the regressor term, which is related to the classical identifiability condition. The case of bounded disturbances (noise of measurements) is analyzed for both algorithms. Simulation results illustrate the feasibility of the proposed algorithms.
\end{abstract}

Index Terms- Time-varying systems, Parameter identification, Finite/Fixed-time.

\section{INTRODUCTION}

$\mathbf{T}$ HE parameter identification problem for different kind of systems has been extensively studied during the last decades. One of the more important reasons is the need for accurate and efficient control for systems. The challenge of providing better models of physical phenomena leads to that the parameter identification problem becomes fundamental in industrial applications. System identification techniques are also used in signal processing applications (such as communications [1], geophysical engineering [2] and mechanical engineering [3]), in nontechnical fields such as biology [4], environmental sciences and econometrics to improve the knowledge on the identified object, prediction and control. The identification theory basically deals with the problem of the efficient extraction of signal and system dynamic properties based on available data measurements. In the literature there exist many methods to identify parameters, and the most popular ones belong to the group of least squares (LS) methods; e.g. non-recursive methods of LS, recursive methods of LS, methods of weighted LS, exponential forgetting with constant forgetting factor, exponential forgetting with variable forgetting factor, etc. There exist also many modifications of the LS methods; e.g. method of generalized LS, method of extended LS, instrumental variables method, and some others like extended Kalman filter, modulating functions methods,

${ }^{\dagger}$ CONACyT - Instituto Tecnológico de La Laguna, División de Estudios de Posgrado e Investigación, Blvd. Revolución y Cuauhtémoc S/N, C.P. 27000, Torreón, Coahuila, México. Email: hriosb@correo.itlalaguna.edu.mx

${ }^{\ddagger}$ Non-A team @ Inria, Parc Scientifique de la Haute Borne, 40 avenue Halley, 59650 Villeneuve d'Ascq, France and CRIStAL (UMR CNRS 9189), Ecole Centrale de Lille, BP 48, Cité Scientifique, 59651 Villeneuve-d'Ascq, France. Emails: denis.efimov@inria.fr; wilfrid.perruquetti@ec-lille.fr

${ }^{\S}$ Eléctrica y Computación, Instituto de Ingeniería, Universidad Nacional Autónoma de México (UNAM), C.P. 04510, México, D.F., Mexico. Emails: JMorenoP@ii.unam.mx; JRuedaE@iingen.unam.mx

* Department of Control Systems and Informatics, Saint Petersburg State University of Information Technologies Mechanics and Optics (ITMO), Kronverkskiy av. 49, Saint Petersburg, 197101, Russia.

${ }^{\star}$ H. Ríos gratefully acknowledge the financial support from CONACyT 270504. This work was also supported in part by HoTSMoCE Inria associate team program, by the Government of Russian Federation (Grant 074-U01) and the Ministry of Education and Science of Russian Federation (Project 14.Z50.31.0031) sub-spaces methods, etc. (see, e.g. [5] and [6]). It is worth mentioning that most of these methods were established for identifying constant parameters. For time-varying parameters, the methods of recursive LS can also be used [6]. For instance, in [7] a non-recursive LS method is proposed for time-varying parameters. In this method, a polynomial approximation, based on Taylor expansion, with a bounded regressor vector is built and used to approximate the time-varying parameters. In [8] a new matrix forgetting factor recursive LS algorithm is proposed for time-varying parameters which satisfy a random walk model assumption. In the framework of adaptive estimation, in [9] a modified version of the LS algorithm is provided to estimate time-varying parameters by means of a polynomial approximation. However, most of these works are only able to follow slowly varying parameters and they can ensure at most exponential or asymptotic convergence to a neighborhood of the real value. In the context of finite-time (FT) convergence [10], a recursive FT convergent algorithm has been presented in [11]. Such an algorithm is a non-linear recursive version of the LS algorithm, where the nonlinear injection terms provide FT convergence since they are designed based on the generalized Super-Twisting Algorithm (STA) [12]. In this line of research, in [13] the STA has also been used for parameter identification of mechanical systems. However, the linearly filtered equivalent output injection signal of the STA is used to obtain the regressor, from which a standard LS recursive algorithm identifies the parameters asymptotically. Other parameter identification methods, using first order sliding-modes, are also based on the reconstruction of the equivalent control signals leading to asymptotic reconstruction algorithms (see, e.g. [14] where an identification scheme is developed for timevarying parameters). A FT and non-recursive LS algorithm is presented by [15] for constant parameters. Such an algorithm is based on adaptive control, it requires to solve matrix valued ordinary differential equations and checking the convertibility of a matrix (persistence of excitation condition) online.

This paper contributes to the development of two parameter identification algorithms that are able to identify time-varying parameters in a finite time and also in a prescribed time (that can be selected a priori), i.e. fixed-time (FxT) [16]; respectively. The convergence proof of the FT identification algorithm is based on a notion of finite-time stability over finite intervals of time, i.e. Short-finite-time (Short-FT) stability [17]; and homogeneity for time-varying systems [18]; a Lyapunov function approach is also given for this algorithm. On the other hand, the convergence proof corresponding to the FxT identification algorithm is also based on a Lyapunov function approach. The results are obtained under injectivity of the regressor term, which is related to the classical identifiability condition. It is worth saying that, to the best of our knowledge, an FxT algorithm for identification of time-varying parameters does not exist in the literature. Additionally, the case of bounded disturbances (noise of measurements) is analyzed for 
both algorithms. Simulation results illustrate the feasibility of the proposed algorithms.

Structure of the paper: The problem statement is presented in the Section II. Some preliminary concepts and results are described in Section III. The FT identification algorithm is presented in Section IV based on time-varying homogeneity and Lyapunov-based approach, respectively. The main result, i.e. the FXT identification algorithm, is proposed in Section V. Some simulation results are depicted in Section VI and some concluding remarks are given in Section VII. Finally, all the proofs are postponed to the Appendix.

Notation: Let $\|q\|$ denote the Euclidean norm of a vector $q \in \mathbb{R}^{n}$, and $\overline{1, n}$ a sequence of integers $1, \ldots, n$. The induced norm for a matrix $Q \in \mathbb{R}^{m \times n}$ is given as $\|Q\|:=$ $\sqrt{\lambda_{\max }\left(Q^{T} Q\right)}=\sigma_{\max }(Q)$, where $\lambda_{\max }$ (respectively, $\left.\lambda_{\min }\right)$ is the maximum (respectively, the minimum) eigenvalue, and $\sigma_{\max }$ is the maximum singular value. For a Lebesgue measurable function $u: \mathbb{R}_{\geq 0} \rightarrow \mathbb{R}^{m}$ define the norm $\|u\|_{\left(t_{0}, t_{1}\right)}:=$

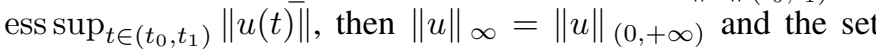
of functions $u$ with the property $\|u\|_{\infty}<+\infty$ is denoted as $\mathcal{L}_{\infty}$. A continuous function $\alpha: \mathbb{R}_{>0} \rightarrow \mathbb{R}_{>0}$ belongs to class $\mathcal{K}$ if it is strictly increasing and $\alpha(0)=0$; it belongs to class $\mathcal{K}_{\infty}$ if it is also unbounded. A continuous function $\beta: \mathbb{R}_{>0} \times \mathbb{R}_{>0} \rightarrow \mathbb{R}_{>0}$ belongs to class $\mathcal{K} \mathcal{L}$ if for each fixed $s, \beta(r, s) \in \mathcal{K}$ with respect to $r$, and for each fixed $r, \beta(r, s)$ is decreasing to zero with respect to $s$.

\section{Problem Statement}

Consider the following time-varying system: ${ }^{1}$

$$
\begin{aligned}
\frac{d \theta(t)}{d t} & =\Theta(\omega t), \\
y(t) & =\Gamma^{T}(\omega t) \theta(t)+\varepsilon(t),
\end{aligned}
$$

where $\theta \in \mathbb{R}^{n}$ and $y \in \mathbb{R}^{m}$ are the unknown parameter vector and the output available for measurements, respectively; while the term $\varepsilon(t) \in \mathbb{R}^{m}$ represents some bounded disturbances (noise of measurements). It is assumed that $\varepsilon$ is a Lebesgue measurable and essentially bounded signal, i.e. $\varepsilon \in \mathcal{L}_{\infty}$. The term $\Gamma: \mathbb{R} \rightarrow \mathbb{R}^{n \times m}$ is a continuous function of time so-called regressor, and $\Theta: \mathbb{R} \rightarrow \mathbb{R}^{n}$ is a uniformly bounded Lebesgue measurable signal. The regressor $\Gamma$ is known, and bounded, whilst $\Theta$ represents the unknown but bounded parameter dynamics and $w$ is the frequency or rate of the time-varying part. The aim of this paper is to identify the time-varying parameter vector $\theta(t)$ in a finite and/or fixed time for the disturbance-free case and provide an ultimate bound for the disturbed case.

\section{PRELIMINARIES}

Consider a time-dependent differential equation [19]:

$$
\frac{d x(t)}{d t}=f(t, x(t)), t \geq t_{0}, t_{0} \in \mathbb{R},
$$

where $x(t) \in \mathbb{R}^{n}$ is the state vector; $f: \mathbb{R} \times \mathbb{R}^{n} \rightarrow \mathbb{R}^{n}$ is a continuous function with respect to $x$ and piece-wise continuous with respect to $t, f(t, 0)=0$ for all $t \in \mathbb{R}$. The solution of the system (3) for an initial condition $x_{0} \in \mathbb{R}^{n}$ at time instant $t_{0} \in \mathbb{R}$ is denoted as $x\left(t, t_{0}, x_{0}\right)$ and defined on some finite time interval $\left[t_{0}, t_{0}+T\right)$.

${ }^{1}$ Note that even when a static relation between the measured output and parameters is considered, such a problem is related with identification of dynamical systems.

\section{A. Stability definitions}

Let $\Omega, \Xi$ be open neighborhoods of the origin in $\mathbb{R}^{n}, 0 \in$ $\Omega \subset \Xi$.

Definition 1. [19], [16] At the steady state $x=0$ the system (3) is said to be

a) Uniformly stable (US) if for any $\epsilon>0$ there is $\delta(\epsilon)$ such that for any $x_{0} \in \Omega$, if $\left\|x_{0}\right\| \leq \delta(\epsilon)$ then $\left\|x\left(t, t_{0}, x_{0}\right)\right\| \leq \epsilon$ for all $t \geq t_{0}$, for any $t_{0} \in \mathbb{R}$;

b) Uniformly finite-time stable (UFTS) if it is US and finitetime converging from $\Omega$, i.e. for any $x_{0} \in \Omega$ there exists $0 \leq T_{x_{0}}<+\infty$ such that $x\left(t, t_{0}, x_{0}\right)=0$ for all $t \geq t_{0}+T_{x_{0}}$, for any $t_{0} \in \mathbb{R}$. The function $T_{0}\left(x_{0}\right)=\inf \left\{T_{x_{0}} \geq 0\right.$ : $\left.x\left(t, t_{0}, x_{0}\right)=0 \forall t \geq t_{0}+T_{x_{0}}\right\}$ is called the settling-time of the system (3).

c) Uniformly fixed-time stable (UFxTS) if it is UFTS and the settling-time function $T_{0}\left(x_{0}\right)$ is bounded, i.e. $\exists T_{\max }>0$ : $T_{0}\left(x_{0}\right) \leq T_{\max }$, for all $x_{0} \in \Omega$ and for any $t_{0} \in \mathbb{R}$.

If $\Omega=\mathbb{R}^{n}$, then $x=0$ is said to be globally US (GUS) / UFTS (GUFTS) / UFxTS (GUFxTS), respectively. In this work a special stability notion will be also used for a compact interval of initial times $t_{0}$, and only on a fixed interval of time [20], [21].

Definition 2. [17] At the steady state $x=0$ the system (3) is said to be

a) Short-time stable (Short-TS) with respect to $\left(\Omega, \Xi, T_{0}, T_{f}\right)$ if for any $x_{0} \in \Omega,\left\|x\left(t, t_{0}, x_{0}\right)\right\| \in \Xi$ for all $t \in\left[t_{0}, T_{f}\right]$ for any $t_{0} \in\left[-T_{0}, T_{0}\right]$;

b) Short-finite-time stable (Short-FTS) with respect to $\left(\Omega, \Xi, T_{0}, T_{f}\right)$ if it is Short-TS with respect to $\left(\Omega, \Xi, T_{0}, T_{f}\right)$ and finite-time converging from $\Omega$ with the convergence time $T_{t_{0}, x_{0}} \leq T_{f}$ for all $x_{0} \in \Omega$ and $t_{0} \in\left[-T_{0}, T_{0}\right]$;

c) Globally short-finite-time stable (GShort-FTS) if for any bounded set $\Omega \subset \mathbb{R}^{n}$ containing the origin there exist a bounded set $\Xi \subset \mathbb{R}^{n}, \Omega \subset \Xi$ and $T_{f}>0$ such that the system is Short-FTS with respect to $\left(\Omega, \Xi, T_{0}, T_{f}\right)$ for any $T_{0}$.

In [20] and [21] the short-time stability is considered only for a fixed initial time instant $t_{0}$. This notion is used here to avoid a confusion with finite-time stability from [22] and [23]; since both concepts of stability are used in this work.

\section{Short-Finite-Time IDEntificAtion Algorithm}

In this section the FT identification algorithm is presented. The convergence to zero of the parameter identification error will be proved based on homogeneity for time-varying systems and Short-FT stability; results introduced previously by [18] and [17]. For simplicity and brevity it is assumed that $t_{0}=0$.

In order to estimate the parameter vector $\theta$, the following nonlinear algorithm can be introduced ${ }^{2}$

$$
\dot{\hat{\theta}}(t)=-K \Gamma(\omega t)\left[\Gamma^{T}(\omega t) \hat{\theta}(t)-\left.y(t)\right|^{\gamma},\right.
$$

where $\left\lceil\left.\cdot\right|^{\gamma}:=|\cdot|^{\gamma} \operatorname{sign}(\cdot)\right.$, with $|\cdot|$ and $\operatorname{sign}(\cdot)$ understood in the component-wise sense, and $\gamma \in[0,1)$; the matrix $K \in$ $\mathbb{R}^{n \times n}$ is symmetric and positive definite, i.e. $K=K^{T}>$ 0 . Define $\sigma_{\Gamma_{\min }}$ and $\sigma_{\Gamma_{\max }}$ as the minimum and maximum singular values of $\Gamma(\omega t)$ for all $t \geq 0$, respectively. Then, let us introduce the following assumption.

\footnotetext{
${ }^{2}$ A similar algorithm was previously presented in [18] for the adaptive state estimation problem; and the discontinuous case, i.e. $\gamma=0$, for time-varying parameter identification without disturbances in [24].
} 
Assumption 1. The regressor term $\Gamma(\omega t)$ is such that $\sigma_{\Gamma_{\min }} \geq$ $\bar{\sigma}>0$ for all $t \geq 0$; while the term $\Theta(\omega t) \in \mathcal{L}_{\infty}$ with $\|\Theta(\omega t)\|_{\infty} \leq k(\omega t) \leq \Lambda$ for all $t \geq 0$, for a known continuous function $k: \mathbb{R} \rightarrow \mathbb{R}_{\geq 0}$ and a known constant $\Lambda>0$.

The assumption $\sigma_{\Gamma_{\min }} \geq \bar{\sigma}>0$ for all $t \geq 0$ implies that $m \geq n$ and it is equivalent to the classic identifiability condition corresponding to the injectivity of the regressor term, i.e. $\operatorname{rank}(\Gamma(\omega t))=n$, for each instant of time $t$.

Let us define the error $\tilde{\theta}(t)=\hat{\theta}(t)-\theta(t)$. Hence, the error dynamics is given by

$$
\dot{\tilde{\theta}}(t)=-K \Gamma(\omega t)\left\lceil\Gamma^{T}(\omega t) \tilde{\theta}(t)-\left.\varepsilon(t)\right|^{\gamma}-\Theta(\omega t) .\right.
$$

In the following, the Short-FT stability statements given by Lemma 3 and Corollary 1 in [17] will be applied, separately; to prove that error dynamics (5), for the disturbance-free case, is GShort-FTS for $\gamma=0$, and globally ultimate bounded for $\gamma \in(0,1)$; while for the disturbed case an ultimate bound is given for any $\gamma \in[0,1)$. All the proofs are described in the Appendix.

\section{A. Homogeneity-based approach: Disturbance-free case}

Let us consider $\varepsilon=0$. Then, the following result is established.

Theorem 1. Let Assumption 1 be satisfied. If $\Theta(0)=0$ and $\varepsilon=0$; then, for any $\rho>0$ and $T_{0}>0$ there exist $\omega_{0}>0$, $\vartheta \geq 1$ and $T_{f}>T_{0}$; such that system (5) with $\omega \in\left[-\omega_{0}, \omega_{0}\right]$, for $\gamma=0$ and $K=K^{T}>0$, is Short-Finite-Time Stable with respect to $\left(B_{\rho}, B_{\vartheta \rho}, T_{0}, T_{f}\right)$.

Remark 1. According to Theorem 1, the Short-FT stability is preserved for a frequency spectrum sufficiently close to zero (see Lemma 3 in [17]).

\section{B. Lyapunov-based approach: Disturbance-free case}

Let us consider $\varepsilon=0$. Thus, based on the statements given by Corollary 1 in [17], the following result is given ${ }^{3}$.

Theorem 2. Let Assumption 1 be satisfied. If there exist $\omega_{0}>$ 0 and $\delta>0$ such that $k$ is a periodic continuous function with $k \in \mathcal{L}_{\varrho, \delta}^{1}$, and $\varrho(s)=\sup _{|t| \leq s} k\left(\omega_{0} t\right)$; then, the system (5), for $\gamma=0$ and $K=K^{T}>0$, is Globally Short-Finite-Time Stable.

Remark 2. From the proof of Theorem 2, if the matrix $K$ is such that $\lambda_{\min }(K)>\left(\lambda_{\max }^{1 / 2}(K) \Lambda / \sigma_{\Gamma_{\min }}\right)^{2 / 3}$, then system (5) is UFTS.

Let us consider the case in which $\gamma \in(0,1)$. Then, the following result is established.

Corollary 1. Let Assumption 1 be satisfied. Then, the system (5), for $\gamma \in(0,1)$ and $K=K^{T}>0$, is globally ultimate bounded, and its trajectories satisfy the following bound

$$
\|\tilde{\theta}(t)\| \leq \sqrt{\frac{\lambda_{\max }(K)}{\lambda_{\min }(K)}} \mu, \forall t \geq T(\tilde{\theta}(0)),
$$

with

$$
\begin{gathered}
\mu=\left(\frac{\Lambda}{\lambda_{\min }(K) \sigma_{\Gamma_{\min }+1}^{\gamma+1}}\right)^{\frac{1}{\gamma}}, \\
T(\tilde{\theta}(0)) \leq \max \left(0, \frac{2\|\tilde{\theta}(0)\| 1-\gamma}{\sigma_{\Gamma_{\min }^{\gamma+1}}^{\gamma+1}(1-\delta) \lambda_{\min }(K)(1-\gamma)}\right),
\end{gathered}
$$

$\delta \in(0,1)$, and $\tilde{\theta}(0) \in \mathbb{R}^{n}$.

${ }^{3}$ The same result has been previously obtained in [24] for the discontinuous algorithm.
Remark 3. The solutions of system (5) enter into the bound (6) at most in a finite time $T(\tilde{\theta}(0))$. According to Definition 2, system (5) is GShort-FTS with respect to the set $\left\{\tilde{\theta} \in \mathbb{R}^{n}:\|\tilde{\theta}\| \leq\right.$ $\left.\sqrt{\lambda_{\max }(K) / \lambda_{\min }(K)} \mu\right\}$.

Remark 4. Corollary 1 shows that the parameter identification error may be reduced according to the choice of the gain $K$ and the parameter $\gamma$ since the size of $\mu$ depends on the value of both of them.

\section{Lyapunov-based approach: Disturbed case}

Let us consider $\varepsilon \neq 0$ and introduce the following assumption.

Assumption 2. The disturbance term $\varepsilon \in \mathcal{L}_{\infty}$ with $\|\varepsilon\|_{\infty} \leq \bar{\varepsilon}$ and a known constant $\bar{\varepsilon}>0$.

Thus, based on the statements given by Corollary 1 , the following result is established.

Corollary 2. Let Assumptions 1 and 2 be satisfied. Then, the system (5), for $\gamma \in[0,1)$ and $K=K^{T}>0$, is globally ultimate bounded, and its trajectories satisfy the following bound

$$
\|\tilde{\theta}(t)\| \leq \sqrt{\frac{\lambda_{\max }(K)}{\lambda_{\min }(K)}} \bar{\mu}, \forall t \geq T(\tilde{\theta}(0)),
$$

with

$$
\begin{gathered}
\bar{\mu}=\max \left[\frac{\bar{\varepsilon}}{\sigma_{\Gamma_{\min }}},\left(\frac{\sigma_{\Gamma_{\max }} m^{\frac{2-\gamma}{4}} \bar{\varepsilon}^{\gamma} \lambda_{\min }(K)+\Lambda}{\lambda_{\min }(K) \sigma_{\Gamma_{\min }^{\gamma+1}} \delta}\right)^{\frac{1}{\gamma}}\right], \\
T(\tilde{\theta}(0)) \leq \max \left(0, \frac{2\|\tilde{\theta}(0)\| 1-\gamma}{\sigma_{\Gamma_{\min }^{\gamma+1}}^{\gamma+\gamma}(1-\delta) \lambda_{\min }(K)(1-\gamma)}\right),
\end{gathered}
$$

$\delta \in(0,1), m \in \mathbb{N}$ the dimension of $y$, and $\tilde{\theta}(0) \in \mathbb{R}^{n}$.

Remark 5. The solutions of system (5) enter into the bound (7) at most in a finite time $T(\tilde{\theta}(0))$. Additionally, system (5) is GShort-FTS with respect to the set $\left\{\tilde{\theta} \in \mathbb{R}^{n}:\|\tilde{\theta}\| \leq \sqrt{\lambda_{\max }(K) / \lambda_{\min }(K)} \bar{\mu}\right\}$. Remark 6. Corollary 2 shows that the parameter identification error converges to a neighborhood of the origin that depends on the magnitude of the noise, i.e. $\bar{\varepsilon}$, the choice of the gain $K$ and the parameter $\gamma$.

\section{FiXed-Time IdENTIFICATION Algorithm}

Let us introduce a modification of the nonlinear algorithm (4), i.e.

$$
\begin{aligned}
\dot{\hat{\theta}}(t)=-K \Gamma(\omega t)\left(\left\lceil\Gamma^{T}(\omega t) \hat{\theta}(t)\right.\right. & -y(t)]^{\gamma_{1}} \\
+ & {\left.\left[\Gamma^{T}(\omega t) \hat{\theta}(t)-y(t)\right]^{\gamma_{2}}\right), }
\end{aligned}
$$

where $\gamma_{1} \in[0,1), \gamma_{2}>1$ and $K=K^{T}>0$. The error dynamics is given as follows

$$
\begin{aligned}
\dot{\tilde{\theta}}(t)=-K \Gamma(\omega t)([ & \left.\Gamma^{T}(\omega t) \tilde{\theta}(t)-\varepsilon(t)\right]^{\gamma_{1}} \\
& +\left\lceil\Gamma^{T}(\omega t) \tilde{\theta}(t)-\left.\varepsilon(t)\right|^{\gamma_{2}}\right)-\Theta(\omega t) .
\end{aligned}
$$

Note that, since $\gamma_{1} \in[0,1)$ and $\gamma_{2}>1$, (9) is not homogeneous. Therefore, only the Lyapunov-based approach is used to prove the FxT stability.

In the following, the FxT stability statements given by Lemma 1 in [16] will be applied to prove that error dynamics (9), for the disturbance-free case, is GFxTS for $\gamma_{1}=0$ and $\gamma_{2}>1$, and globally ultimate bounded for $\gamma_{1} \in(0,1)$ and $\gamma_{2}>1$; while for the disturbed case an ultimate bound is given for any $\gamma_{1} \in(0,1)$ and $\gamma_{2}>1$. All the proofs are described in the Appendix. 
A. Lyapunov-based approach: Disturbance-free case

Let us consider $\varepsilon=0$. Based on the statements given by Lemma 1 in [16], the following result is established.

Theorem 3. Let Assumption 1 be satisfied. If the following conditions hold:

$$
\text { 1) } \lambda_{\min }(K)>\max \left(\bar{\lambda}_{1}, \bar{\lambda}_{2}\right) \text {, with }
$$$$
\bar{\lambda}_{1}=\left(\frac{n^{\frac{\gamma_{2}-1}{2\left(\gamma_{2}+1\right)}} \sqrt{\lambda_{\max }(K)} \Lambda}{2^{\frac{\gamma_{2}}{2}} \sigma_{\Gamma_{\min }}^{\gamma_{2}+1}}\right)^{\frac{2}{\gamma_{2}+3}}, \bar{\lambda}_{2}=\left(\frac{\sqrt{\lambda_{\max }(K)} \Lambda}{\sigma_{\Gamma_{\min }}}\right)^{\frac{2}{3}}
$$

2) $\gamma_{1}=0$ and $\gamma_{2}>1$;

then, the system (9) is Globally Fixed-Time Stable with settling time

$$
T \leq \frac{2\left(\alpha\left(\gamma_{2}+1\right)+\beta\right)}{\alpha \beta\left(\gamma_{2}+1\right)},
$$

for all $\tilde{\theta}(0) \in \mathbb{R}^{n}$ and

$$
\begin{gathered}
\alpha=\frac{\sigma_{\Gamma_{\min }}^{\gamma_{2}+1}\left(2 \lambda_{\min }(K)\right)^{\frac{\gamma_{2}+1}{2}}}{n^{\frac{\gamma_{2}-1}{2\left(\gamma_{2}+1\right)}}}-\frac{\sqrt{2 \lambda_{\max }(K)}}{\lambda_{\min }(K)} \Lambda, \\
\beta=\sigma_{\Gamma_{\min }}\left(2 \lambda_{\min }(K)\right)^{\frac{1}{2}}-\frac{\sqrt{2 \lambda_{\max }(K)}}{\lambda_{\min }(K)} \Lambda .
\end{gathered}
$$

Remark 7. The solutions of the error dynamics (9), for $\gamma_{1}=0$ and $\gamma_{2}>1$, go to zero at most in a fixed time $T$ that is independent of $\theta(0)$.

Let us consider the case in which $\gamma_{1} \in(0,1)$ and $\gamma_{2}>$

1. Then, based on the statements given by Corollary 1 and Theorem 3, the following result is established.

Corollary 3. Let Assumption 1 be satisfied. If the following conditions hold:

1) $\lambda_{\min }(K)>\bar{\lambda}_{1}$;

2) $\gamma_{1} \in(0,1)$ and $\gamma_{2}>1$;

then, the system (9) is globally ultimate bounded, and its trajectories satisfy the following bound

$$
\|\tilde{\theta}(t)\| \leq \sqrt{\frac{\lambda_{\max }(K)}{\lambda_{\min }(K)}} \min \left(\sqrt{2 \lambda_{\max }(K)}, \mu_{f}\right), \forall t \geq T(\tilde{\theta}(0)),
$$

with

$$
\mu_{f}=\left(\frac{\Lambda}{\lambda_{\min }(K) \sigma_{\Gamma_{\min }}^{\gamma_{1}+1} \delta}\right)^{\frac{1}{\gamma_{1}}}, T \leq \max \left(0, \frac{2}{\alpha\left(\gamma_{2}+1\right)}\right),
$$

$\delta \in(0,1)$, and all $\tilde{\theta}(0) \in \mathbb{R}^{n}$.

Remark 8. The solutions of (9), for $\gamma_{1} \in(0,1)$ and $\gamma_{2}>1$, enter into the bound (10) at most in a fixed time $T$ that is independent of $\tilde{\theta}(0)$. In this sense, the Algorithm described by (8) may possess a faster rate of convergence to the bound (10) than the Algorithm given by (4).

Remark 9. Corollary 3 shows that the parameter identification error could be adjusted according to the choice of the gain $K$ and the parameter $\gamma_{1}$.

\section{B. Lyapunov-based approach: Disturbed case}

Let us consider $\varepsilon \neq 0$. Thus, based on the statements given by Corollary 3 , the following result is established.

Corollary 4. Let Assumptions 1 and 2 be satisfied. If the following conditions hold:

1) $\lambda_{\max }(K)<\bar{\lambda}_{3}$, with

$$
\bar{\lambda}_{3}=\left(\frac{2^{\frac{2-\gamma_{2}}{2}} \sigma_{\Gamma_{\min }}^{\gamma_{2}+1}}{n^{\frac{\gamma_{2}-1}{2\left(\gamma_{2}+1\right)}\left(\sigma_{\Gamma_{\max }}\left(m \frac{2-\gamma_{1}}{4} \bar{\varepsilon} \gamma_{1}+m \frac{2-\gamma_{2}}{4} \bar{\varepsilon} \gamma_{2}\right) \lambda_{\min }(K)+\Lambda\right)}}\right)^{\frac{1}{2}}
$$

2) $\gamma_{1} \in[0,1)$, and $\gamma_{2}>1$;

then, the system (9) is globally ultimate bounded, and its trajectories satisfy the following bound

$$
\|\tilde{\theta}(t)\| \leq \sqrt{\frac{\lambda_{\max }(K)}{\lambda_{\min }(K)}} \min \left(\sqrt{2 \lambda_{\max }(K)}, \bar{\mu}_{f}\right), \forall t \geq T,
$$

with

$$
\begin{gathered}
\bar{\mu}_{f}=\max \left[\frac{\bar{\varepsilon}}{\sigma_{\Gamma_{\min }}},\left(\frac{\sigma_{\Gamma_{\max }}\left(m \frac{2-\gamma_{1}}{4} \bar{\varepsilon}^{\gamma_{1}}+m \frac{2-\gamma_{2}}{4} \bar{\varepsilon}_{2}\right) \lambda_{\min }(K)+\Lambda}{\lambda_{\min }(K) \sigma_{\Gamma_{\min } \gamma_{1}+1} \delta}\right)^{\frac{1}{\gamma_{1}}}\right], \\
T \leq \max \left(0, \frac{2}{\bar{\alpha}\left(\gamma_{2}+1\right)}\right),
\end{gathered}
$$

$\delta \in(0,1), m \in \mathbb{N}$ the dimension of $y$, all $\tilde{\theta}(0) \in \mathbb{R}^{n}$, and

$$
\bar{\alpha}=\frac{2^{\frac{3-\gamma_{2}}{2}} \sigma_{\Gamma_{\min }}^{\gamma_{2}+1}\left(\lambda_{\min }(K)\right)^{\frac{\gamma_{2}+1}{2}}}{n^{\frac{\gamma_{2}-1}{2\left(\gamma_{2}+1\right)}}}-\frac{\sqrt{2 \lambda_{\max }(K)}}{\lambda_{\min }(K)} \Lambda .
$$

Remark 10. The solutions of (9) enter into the bound (11) at most in a fixed time $T$.

Remark 11. Corollary 4 shows that the parameter identification error converges to a neighborhood of the origin that depends on the magnitude of the noise, i.e. $\bar{\varepsilon}$, the choice of the gain $K$ and the parameters $\gamma_{1}$ and $\gamma_{2}$; in a fixed time $T$.

\section{Simulation Results}

\section{A. Automatic throttle valve actuator}

Consider the behavior of an automatic throttle valve actuator [6]. The DC motor is described by

$$
\begin{aligned}
U(t) & =R(t) i(t)+\psi(t) \omega(t), \\
M_{e l}(t) & =\psi(t) i(t),
\end{aligned}
$$

where $U$ is the armature voltage, $M_{e l}$ is an electrical time variable, $R$ and $\psi$ are the unknown time-varying armature resistance and magnetic flux linkage, respectively; while $i$ and $\omega$ are the armature phase current and motor angular speed, respectively. The mechanical part is modeled as

$$
\begin{aligned}
J \dot{\omega}(t) & =\psi(t) i(t)-\frac{1}{v}\left(c_{s} \varphi(t)+f_{p}+f_{c}\lceil\omega(t)\rfloor^{0}+f_{s} \frac{\omega(t)}{v}\right), \\
\dot{\varphi}(t) & =\frac{\omega(t)}{v}, \omega(0)=0, \varphi(0)=0,
\end{aligned}
$$

where $J$ is the inertia, $v$ is the gear ratio, $c_{s}$ and $f_{p}$ are the spring constant and pretension, respectively; $f_{c}$ is the Coulomb friction torque, $f_{s}$ is the viscous friction torque, and $\varphi$ is the angular throttle position. The model for the parameter identification is then given as

$$
y(t)=\Gamma^{T}(t) \theta(t)+\varepsilon(t),
$$

where $y(t):=\left[U(t), M_{e l}(t)\right]^{T}$ is the measured output, $\theta(t):=$ $[R(t), \psi(t)]^{T}$ is the unknown time-varying parameter vector, $\varepsilon(t)$ represents the disturbances, and

$$
\Gamma(t)=\left[\begin{array}{cc}
i(t) & 0 \\
\omega(t) & i(t)
\end{array}\right] .
$$

The parameters of the model are given in Table I.

Table I

PARAMETERS OF THE AUTOMATIC THROTTLE VALVE ACTUATOR.

\begin{tabular}{|c|c|}
\hline Parameter & Value \\
\hline \hline$i(t)$ & $0.5 \sin (2 \pi f t)[A]$ \\
\hline$f$ & $60[\mathrm{~Hz}]$ \\
\hline$R(t)$ & $\cos (0.2 t)[\Omega]$ \\
\hline$\psi(t)$ & $\sin (0.1 \mathrm{t})[\mathrm{Vs}]$ \\
\hline$J$ & $0.011\left[\mathrm{kgm}^{2}\right]$ \\
\hline$v$ & 16.42 \\
\hline$c_{s}$ & $0.01[\mathrm{~N} / \mathrm{m}]$ \\
\hline$f_{p}$ & $0.7[\mathrm{Nm}]$ \\
\hline$f_{c}$ & $1.0[\mathrm{Nm}]$ \\
\hline$f_{s}$ & $0.0037[\mathrm{Nm}]$ \\
\hline
\end{tabular}


It is easy to show that the given example satisfies Assumption 1 with $\sigma_{\Gamma_{\min }}=0.5147$ and $\Lambda=0.3$. The simulations have been done in Matlab Simulink with the Euler discretization method and sample time equal to 0.001. The FT and FxT algorithms, i.e. (4) and (8), respectively; are implemented for the disturbance-free case, i.e. $\varepsilon(t)=0$, with $\gamma=0$ for the FT algorithm, and $\gamma_{1}=0, \gamma_{2}=1.5$ for the FxT algorithm; both of them with $K=3 I$, and different initial conditions. Note that this value of $K$ satisfies the conditions of Theorem 2, i.e. $K=K^{T}>0$, and also the conditions of Theorem 3, i.e. $\lambda_{\min }(K)>\max \left(\bar{\lambda}_{1}, \bar{\lambda}_{2}\right)$ with $\bar{\lambda}_{1}=0.0845$ and $\bar{\lambda}_{2}=0.7481$. The results are depicted by Figs. 1 and 2 . The results illustrate the statements given by Theorem 2 and 3, i.e. FT and FxT convergence, respectively.
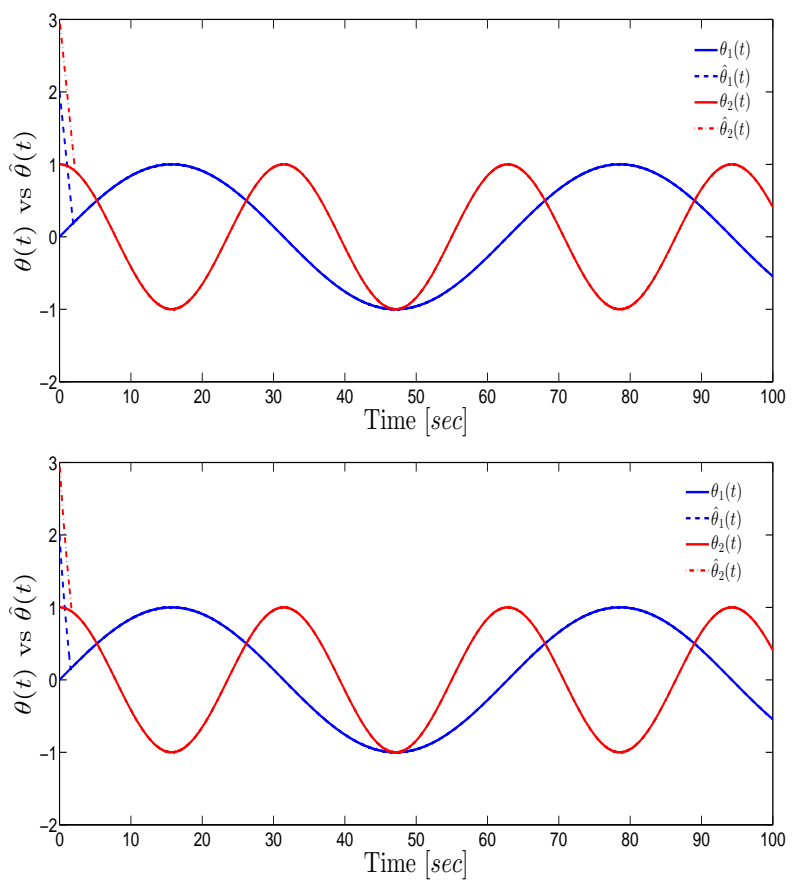

Figure 1. Parameter Identification Noise-Free. The top graph shows the parameter identification for the case $\gamma=0$ (FT algorithm) while the bottom graph for the case $\gamma_{1}=0$ and $\gamma_{2}=1.5$ (FxT algorithm), with $\theta(0)=$ $(0,1)^{T}$ and $\hat{\theta}(0)=(2,3)^{T}$.

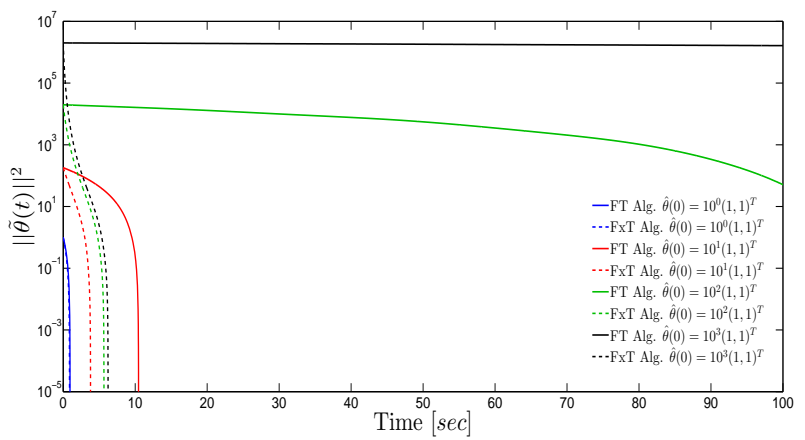

Figure 2. Parameter Identification Error Disturbance-Free. The graph shows that for the FT algorithm (solid lines) the convergence time increases when the initial conditions of the error dynamics also increases, while the FxT algorithm depicts in addition to a faster rate of convergence a bounded settling time $T_{0}(\tilde{\theta}(0))$ for any initial condition $\tilde{\theta}(0) \in \mathbb{R}^{2}$.

For the disturbed case, the FT and FxT algorithms are designed in the same way as in the previous simulation taking into account that the disturbance $\varepsilon(t):=\left[\varepsilon_{1}(t), \varepsilon_{1}(t)\right]^{T}$ is given by a bounded continuous signal such that $\left\|\varepsilon_{1}\right\|_{\infty} \leq$ $\bar{\varepsilon}=2$. Note that the value $K=3 I$ satisfies the conditions of Corollary 2, i.e. $K=K^{T}>0$, and also the conditions of Corollary 4, i.e. $\lambda_{\max }(K)<\bar{\lambda}_{3}=3.5230$. The results are depicted by Fig. 3. The results illustrate the statements given by Corollary 2 and 4, i.e. global ultimate boundedness.

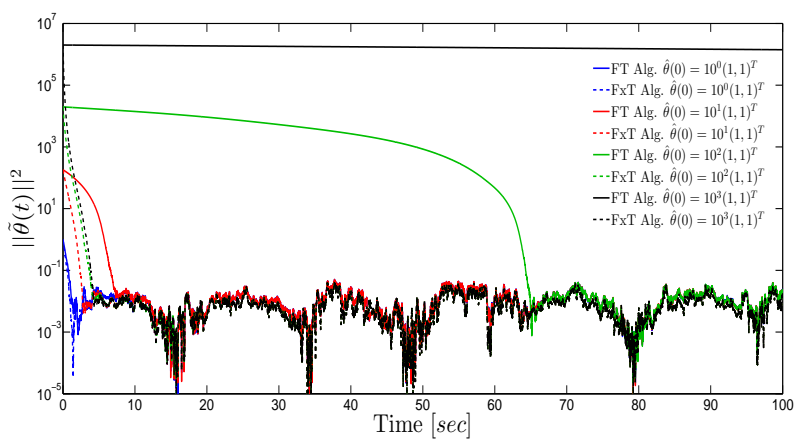

Figure 3. Parameter Identification Error - Disturbance Case

Now, the algorithms given in (4) and (8), are implemented with $K=3 I$ and different values of $\gamma, \gamma_{1} \in(0,1)$, respectively; and $\gamma_{2} \in[1.5,3.0]$ for the algorithm given by (8) and the disturbance case. The parameter identification error for both algorithms is depicted by Fig. 4. The results illustrate the statements given by Remarks 6 and 11, respectively.
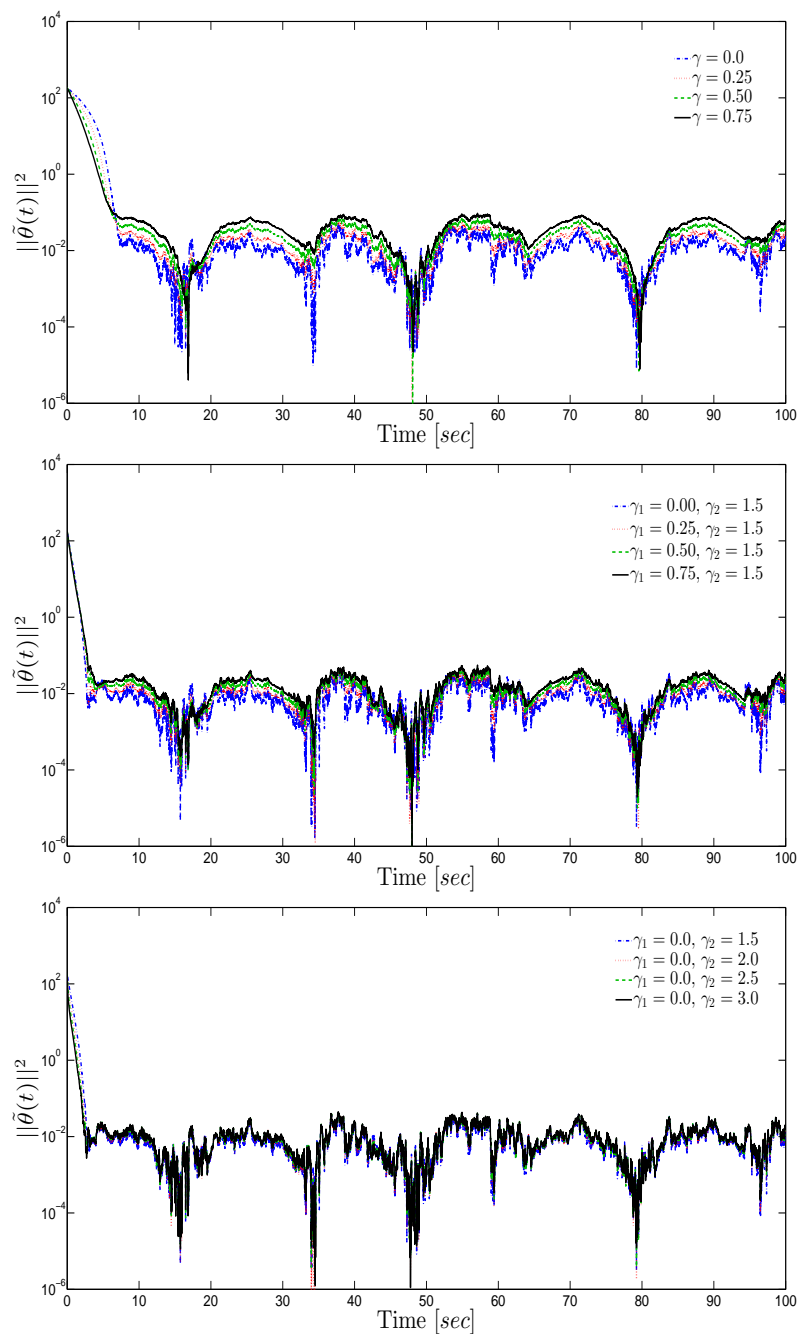

Figure 4. Parameter Identification Error Disturbance-Case. The top graph shows the parameter identification error for different values of $\gamma \in(0,1)$ (algorithm (4)), the bottom graph for the case $\gamma_{1}=0$ and $\gamma_{2} \in[1.5,3.0]$, while the middle graph for the case $\gamma_{1} \in(0,1)$ and $\gamma_{2}=1.5$ (algorithm $(8))$, with $\theta(0)=(0,1)^{T}$ and $\hat{\theta}(0)=(10,10)^{T}$. 


\section{B. Example with relaxed Assumption 1}

Let us consider another example, i.e.

$$
\begin{aligned}
& \dot{\theta}_{1}(t)=\sin (3.5 t)+\cos (2 t), \theta_{1}(0)=2, \\
& \dot{\theta}_{2}(t)=\sin (0.1 t)+\cos (3 t), \theta_{2}(0)=2,
\end{aligned}
$$

with the following structure for the regressor

$$
\Gamma(t)=\left[\begin{array}{ccc}
0.2 \cos (50 t) & -0.1 \cos (50 t) & 0.2 \cos (50 t) \\
0.1 & -0.05 & 0.1
\end{array}\right] .
$$

For this example $\operatorname{rank}(\Gamma(t))=1$ for all $t$, i.e. Assumption 1 is not satisfied, and the injectivity condition of the regressor term does not hold for each instant of time. The FxT algorithm (8) is implemented with $\gamma_{1}=0, \gamma_{2}=1.5, K=15 I$, and initial conditions $\hat{\theta}(0)=(1,1)^{T}$; and for comparison purpose the Pseudo-inverse solution (least-square) is also implemented, i.e. $\hat{\theta}(t)=\left(\Gamma(t) \Gamma^{T}(t)\right)^{-1} \Gamma(t) y$. The results are depicted by Fig. 5 for the disturbance-free case, i.e. $\varepsilon(t)=0$.
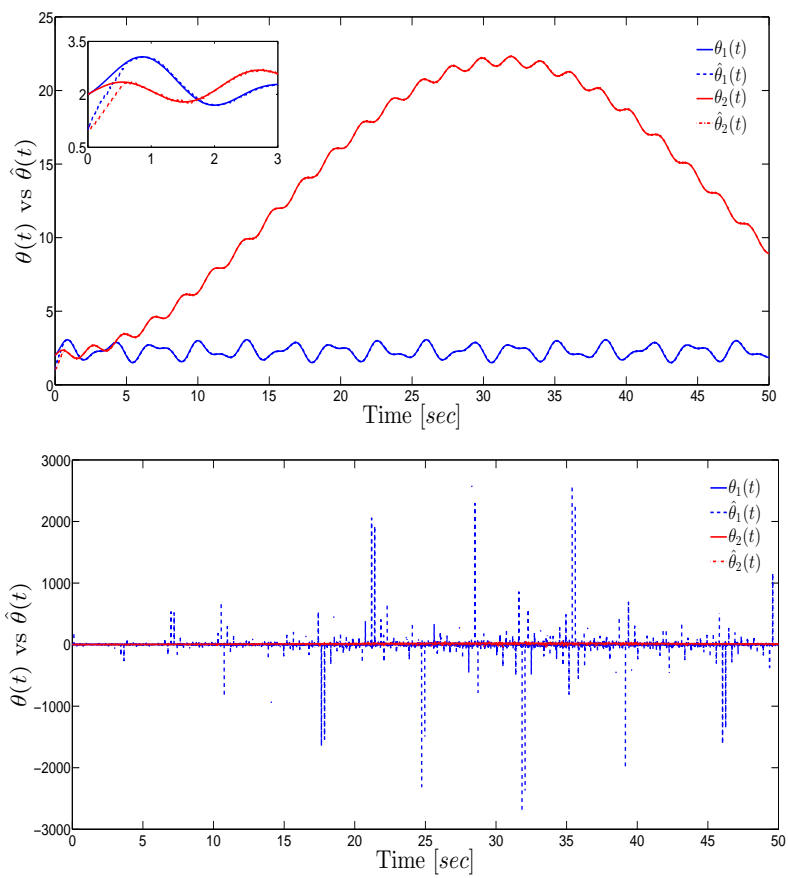

Figure 5. FxT Algorithm vs Pseudo-inverse Solution Disturbance-Free. The top graph shows the parameter identification for the FxT algorithm while the bottom one depicts the solution given by the least-square method.

Despite that Assumption 1 does not hold, the proposed FxT Algorithm works while the conventional inversion algorithm fails to provide an estimation in this case. The explanations of this phenomenon lies in the persistence of excitation condition which is a subject of future research.

\section{CONCLUSIONS}

Two identification algorithms, i.e. FT and FxT algorithms, are proposed that are able to identify time-varying parameters in a finite-time and also in a prescribed time, respectively. The convergence proof of the FT identification algorithm is based on Short-FT stability and homogeneity for time-varying systems; and also a Lyapunov-based approach is given for this algorithm. On the other hand, the convergence proof of the FxT algorithm is based on a Lyapunov-based approach. The results are obtained under injectivity of the regressor term, which is related to the classical identifiability condition. It is worth saying that, to the best of our knowledge, an FxT algorithm to identify time-varying parameters does not exist in the literature. Additionally, the case of bounded disturbances (noise of measurements) is analyzed for both algorithms. Simulation results depict the feasibility of the proposed algorithms. The persistence of excitation properties are in the scope of future research.

\section{APPENDIX}

Let us introduce the following class of functions for $\varrho \in \mathcal{K}$ and $\delta>0$ :

$$
\begin{aligned}
& \mathcal{L}_{\varrho, \delta}^{m}=\left\{d: \mathbb{R} \rightarrow \mathbb{R}^{m}:\|d(s)\| \leq \varrho(s) \forall s \geq 0 ;\right. \\
& \left.\quad \exists \tau>0: d(s)=0, \forall|s| \geq \tau ; \max \left\{\|d\|_{1},\|d\|_{\infty}\right\} \leq \delta\right\},
\end{aligned}
$$

where $\|d\|_{1}=\int_{-\infty}^{+\infty}\|d(t)\| d t,\|d\|_{\infty}=\sup _{t \in \mathbb{R}}\|d(t)\|$.

Proof of Theorem 1: Let us apply the statements given in Lemma 3 in [17] in order to prove that the system (5) is Short-FTS:

1. System (5) is $r$-homogeneous with degree $\nu=-1$ for $\left(r_{1}, r_{2}, \ldots, r_{n}\right)=(1,1, \ldots, 1)$ and $\gamma=0$.

2. Let us prove that system (5) is GAS for $\omega=0$. Assume that $\Theta(0)=0$ and define $\Gamma_{0}=\Gamma(0)$. Then, let us consider the following candidate Lyapunov function

$$
V(t)=\frac{1}{2} \tilde{\theta}^{T} K^{-1} \tilde{\theta} .
$$

The time derivative along the trajectories of system (5) is given as follows

$$
\dot{V}(t)=\tilde{\theta}^{T} K^{-1}\left(-K \Gamma_{0}\left\lceil\Gamma_{0}^{T} \tilde{\theta}\right\rfloor^{\gamma}\right)=-\tilde{\theta} \Gamma_{0}\left\lceil\Gamma_{0}^{T} \tilde{\theta}\right]^{\gamma} .
$$

Note that

$$
\tilde{\theta}^{T} \Gamma_{0}\left\lceil\Gamma_{0}^{T} \tilde{\theta}\right\rfloor^{\gamma}=\sum_{i=1}^{m}\left|\left(\tilde{\theta}^{T} \Gamma_{0}\right)_{i}\right|^{\gamma+1}=\left\|\tilde{\theta}^{T} \Gamma_{0}\right\| \begin{gathered}
\gamma+1 \\
\gamma+1
\end{gathered},
$$

and since $\left\|\tilde{\theta}^{T} \Gamma_{0}\right\| \leq\left\|\tilde{\theta}^{T} \Gamma_{0}\right\|_{\gamma+1}$ holds for all $2>\gamma+1>0, \dot{V}$ may be bounded as follows

$$
\begin{aligned}
\dot{V}(t) & \leq-\left\|\tilde{\theta}^{T} \Gamma_{0}\right\| \begin{array}{l}
\gamma+1 \\
\gamma+1
\end{array} \leq-\left\|\tilde{\theta}^{T} \Gamma_{0}\right\| \|^{\gamma+1}, \\
& \leq-\left(\tilde{\theta}^{T} \Gamma_{0} \Gamma_{0}^{T} \tilde{\theta}\right)^{\frac{\gamma+1}{2}} \leq-\sigma_{\Gamma_{\min }^{\gamma+1}}^{\gamma+1}\|\tilde{\theta}\|^{\gamma+1} .
\end{aligned}
$$

Hence, $\dot{V}$ is negative definite and thus, GAS is concluded for $\omega=0$. 3. Since $\Gamma$ is a continuous function of time and $\Theta$ is a uniformly bounded Lebesgue measurable signal, Assumption 1 in in [17] is satisfied for all $\gamma \in[0,1)$.

Therefore, based on Lemma 3 in [17], there exist $\omega_{0}>0, \vartheta \geq 1$ and $T_{f}>T^{0}$; such that system (5) with $\omega \in\left[-\omega_{0}, \omega_{0}\right], \gamma=0, \varepsilon=0$ and $K=K^{T}>0$ is Short-FTS with respect to $\left(B_{\rho}, B_{\vartheta \rho}, T^{0}, T_{f}\right)$.

Proof of Theorem 2: Let us consider the Lyapunov function (12) which satisfies the following inequalities

$$
\begin{gathered}
c_{1}^{-1}\|\tilde{\theta}\|^{2} \leq V \leq c_{2}^{-1}\|\tilde{\theta}\|^{2}, \\
c_{1}^{-\frac{\gamma+1}{2}}\|\tilde{\theta}\|^{\gamma+1} \leq V^{\frac{\gamma+1}{2}} \leq c_{2}^{-\frac{\gamma+1}{2}}\|\tilde{\theta}\| \gamma{ }^{\gamma+1},
\end{gathered}
$$

where $c_{1}=2 \lambda_{\max }(K)$ and $c_{2}=2 \lambda_{\min }(K)$. The function $V$ is positive definite, radially unbounded, and continuously differentiable with its time derivative satisfying

$$
\begin{aligned}
\dot{V}(t) & =\tilde{\theta}^{T} K^{-1}\left(-K \Gamma\left[\Gamma^{T} \tilde{\theta}\right]^{\gamma}-\Theta(\omega t)\right), \\
& \leq-\tilde{\theta}^{T} \Gamma\left\lceil\Gamma^{T} \tilde{\theta}\right]^{\gamma}+\frac{\|\tilde{\theta}\|\|\Theta(\omega t)\|_{\infty}}{\lambda_{\min }(K)} .
\end{aligned}
$$

Then, recalling that $\tilde{\theta}^{T} \Gamma\left\lceil\Gamma^{T} \tilde{\theta}\right]^{\gamma}=\left\|\tilde{\theta}^{T} \Gamma\right\| \begin{aligned} & \gamma+1 \\ & \gamma+1\end{aligned}$, and since $\left\|\tilde{\theta}^{T} \Gamma\right\| \leq\left\|\tilde{\theta}^{T} \Gamma\right\|_{\gamma+1}$ holds for all $2>\gamma+1>0$, and $\|\Theta(\omega t)\|_{\infty} \leq k(\omega t), \dot{V}$ may be bounded as follows

$$
\begin{aligned}
\dot{V}(t) & \leq-\left\|\tilde{\theta}^{T} \Gamma\right\| \gamma+1+\frac{k(\omega t)}{\lambda_{\min }(K)}\|\tilde{\theta}\|, \\
& \leq-\sigma_{\Gamma_{\min }^{\gamma+1}}^{\gamma}\|\tilde{\theta}\| \gamma+1+\frac{k(\omega t)}{\lambda_{\min }(K)}\|\tilde{\theta}\|, \\
& \leq-\sigma_{\Gamma_{\min }^{\gamma+1}} c_{2}^{\frac{\gamma+1}{2}} V^{\frac{\gamma+1}{2}}(t)+\frac{2 \sqrt{c_{1}}}{c_{2}} k(\omega t) V^{\frac{1}{2}}(t) .
\end{aligned}
$$


Let us assume that $\gamma=0$. Therefore, from (15) and (13), it follows that

$$
\dot{V}(t) \leq-\sigma_{\Gamma_{\min }} \sqrt{c_{2}} V^{\frac{1}{2}}(t)+\frac{2 \sqrt{c_{1}}}{c_{2}} k(\omega t) V^{\frac{1}{2}}(t) .
$$

Applying Corollary 1 in [17], with $\alpha=\sigma_{\Gamma_{\min }} \sqrt{c_{2}}, \eta=0.5$, and assuming that $k$ is periodic, and such that $k \in \mathcal{L}_{\rho, \delta}^{1}$, with $\varrho(s)=$ $\sup _{|t| \leq s} k\left(\omega_{0} t\right)$, one can conclude that the error (5) is GShort-FTS.

Proof of Corollary 1: From (15) and the fact that $\|\Theta(\omega t)\|_{\infty} \leq \Lambda$, for all $t \in \mathbb{R}$, it follows that

$$
\begin{aligned}
\dot{V}(t) & \leq-\sigma_{\Gamma_{\min }}^{\gamma+1}\|\tilde{\theta}\|^{\gamma+1}+\frac{\Lambda}{\lambda_{\min }(K)}\|\tilde{\theta}\|, \\
& \leq-\sigma_{\Gamma_{\min }}^{\gamma+1}(1-\delta)\|\tilde{\theta}\|^{\gamma+1}-\sigma_{\Gamma_{\min }}^{\gamma+1} \delta\|\tilde{\theta}\|^{\gamma+1}+\frac{\Lambda}{\lambda_{\min }(K)}\|\tilde{\theta}\|,
\end{aligned}
$$

where $\delta \in(0,1)$. Then,

$$
\dot{V}(t) \leq-\sigma_{\Gamma_{\min }}^{\gamma+1}(1-\delta)\|\tilde{\theta}\|^{\gamma+1}, \forall\|\tilde{\theta}\| \geq \mu .
$$

From (16), it follows that

$$
\dot{V}(t) \leq-\sigma_{\Gamma_{\min }}^{\gamma+1}(1-\delta) c_{2}^{\frac{\gamma+1}{2}} V^{\frac{\gamma+1}{2}}(t),
$$

and by the comparison principle (see, e.g. [19]), one obtains

$$
V(t) \leq\left(V^{\frac{1-\gamma}{2}}(0)-\frac{\sigma_{\Gamma_{\min }}^{\gamma+1}(1-\delta) c_{2}^{\frac{\gamma+1}{2}}(1-\gamma)}{2} t\right)^{\frac{2}{1-\gamma}},
$$

then, the last inequality ensures that $\tilde{\theta}$ satisfies the following bound

$$
\begin{aligned}
& \|\tilde{\theta}(t)\| \leq \sqrt{c_{1}} \times \\
& \left(c_{2}^{\frac{\gamma-1}{2}}\|\tilde{\theta}(0)\|^{1-\gamma}-\frac{\sigma_{\Gamma_{\min }}^{\gamma+1}(1-\delta) c_{2}^{\frac{\gamma+1}{2}}(1-\gamma)}{2} t\right)^{\frac{1}{1-\gamma}},
\end{aligned}
$$

for all $t<T(\tilde{\theta}(0))$; while for all $t \geq T(\tilde{\theta}(0))$, from (13), it is obtained that $\tilde{\theta}$ is bounded as in (6), i.e. $\|\tilde{\theta}\| \leq \sqrt{\lambda_{\max }(K) / \lambda_{\min }(K)} \mu$. Note that (6) and (17) hold for any $\tilde{\theta}(0) \in \mathbb{R}^{n}$, with no restriction on how large $\mu$ is.

Hence, it is concluded that the solutions of system (5) are globally ultimate bounded with its trajectories satisfying the bound given by (6).

Proof of Corollary 2: Let us consider the Lyapunov function (12) which satisfies the inequalities (13) and (14). The time derivative of $V$ satisfies

$$
\begin{aligned}
\dot{V}(t) & =\tilde{\theta}^{T} K^{-1}\left(-K \Gamma\left\lceil\Gamma^{T} \tilde{\theta}-\varepsilon\right]^{\gamma}-\Theta(\omega t)\right), \\
& \leq-\tilde{\theta}^{T} \Gamma\left[\Gamma^{T} \tilde{\theta}-\varepsilon\right]^{\gamma}+\frac{\|\tilde{\theta}\| \Lambda}{\lambda_{\min }(K)} .
\end{aligned}
$$

Consider, in the component-wise sense, that $\left|\left(\Gamma^{T} \tilde{\theta}\right)_{i}\right| \geq\left|\varepsilon_{i}\right|$, for all $i=\overline{1, m}$. Therefore, $\operatorname{sign}\left(\Gamma^{T} \tilde{\theta}-\varepsilon\right)=\operatorname{sign}\left(\Gamma^{T} \tilde{\theta}\right)$ is implied. Then, for any $x, \bar{x} \in \mathbb{R}$ and $\gamma \in[0,1)$, the inequality $|x+\bar{x}|^{\gamma} \leq|x|^{\gamma}+|\bar{x}|^{\gamma}$ holds [25]. Hence, defining $x=\left(\Gamma^{T} \tilde{\theta}\right)_{i}-\varepsilon_{i}$ and $\bar{x}=\varepsilon_{i}$, it follows that for all $i=\overline{1, n}$

$$
\begin{aligned}
\left|\left(\Gamma^{T} \tilde{\theta}\right)_{i}\right|^{\gamma} & =\left|\left(\Gamma^{T} \tilde{\theta}\right)_{i}-\varepsilon_{i}+\varepsilon_{i}\right|^{\gamma} \leq\left|\left(\Gamma^{T} \tilde{\theta}\right)_{i}-\varepsilon_{i}\right|^{\gamma}+\left|\varepsilon_{i}\right|^{\gamma}, \\
& \Rightarrow\left|\left(\Gamma^{T} \tilde{\theta}\right)_{i}\right|^{\gamma}-\left|\varepsilon_{i}\right|^{\gamma} \leq\left|\left(\Gamma^{T} \tilde{\theta}\right)_{i}-\varepsilon_{i}\right|^{\gamma},
\end{aligned}
$$

and then, in the component-wise sense, one gets that

$$
-\left|\Gamma^{T} \tilde{\theta}-\varepsilon\right|^{\gamma} \leq-\left|\Gamma^{T} \tilde{\theta}\right|^{\gamma}+|\varepsilon|^{\gamma},
$$

holds for any $\gamma \in[0,1)$. Applying (18), one obtains that $\dot{V}$ is upper bounded as follows

$$
\dot{V}(t) \leq-\tilde{\theta}^{T} \Gamma\left(\left\lceil\Gamma^{T} \tilde{\theta}\right]^{\gamma}-|\varepsilon|^{\gamma} \operatorname{sign}\left(\Gamma^{T} \tilde{\theta}\right)\right)+\frac{\|\tilde{\theta}\| \Lambda}{\lambda_{\min }(K)} .
$$

Recall that $[\cdot]^{\gamma},|\cdot|$ and $\operatorname{sign}(\cdot)$ are understood in the componentwise sense, i.e.

$$
\begin{gathered}
\left\lceil\Gamma^{T} \tilde{\theta}\right\rfloor^{\gamma}=\left(\begin{array}{c}
\left|\left(\Gamma^{T} \tilde{\theta}\right)_{1}\right|^{\gamma} \operatorname{sign}\left(\left(\Gamma^{T} \tilde{\theta}\right)_{1}\right) \\
\left|\left(\Gamma^{T} \tilde{\theta}\right)_{2}\right|^{\gamma} \operatorname{sign}\left(\left(\Gamma^{T} \tilde{\theta}\right)_{2}\right) \\
\vdots \\
\left|\left(\Gamma^{T} \tilde{\theta}\right)_{m}\right|^{\gamma} \operatorname{sign}\left(\left(\Gamma^{T} \tilde{\theta}\right)_{m}\right)
\end{array}\right), \\
|\varepsilon|^{\gamma} \operatorname{sign}\left(\Gamma^{T} \tilde{\theta}\right)=\left(\begin{array}{c}
\left.\left|\varepsilon_{1}\right|\right|^{\gamma} \operatorname{sign}\left(\left(\Gamma^{T} \tilde{\theta}\right)_{1}\right) \\
\left|\varepsilon_{2}\right|^{\gamma} \operatorname{sign}\left(\left(\Gamma^{T} \tilde{\theta}\right)_{2}\right) \\
\vdots \\
\left|\varepsilon_{m}\right|^{\gamma} \operatorname{sign}\left(\left(\Gamma^{T} \tilde{\theta}\right)_{m}\right)
\end{array}\right) .
\end{gathered}
$$

Then, it follows that

$$
\begin{array}{r}
\dot{V}(t) \leq-\sigma_{\Gamma_{\min }+1}^{\gamma+\hat{\theta}}\|\|^{\gamma+1}+\tilde{\theta}^{T} \Gamma\left(|\varepsilon|^{\gamma} \operatorname{sign}\left(\Gamma^{T} \tilde{\theta}\right)\right)+\frac{\|\tilde{\theta}\| \Lambda}{\lambda_{\min }(K)}, \\
\leq-\sigma_{\Gamma_{\min }+1}^{\gamma+1}\|\tilde{\theta}\|\left\|^{\gamma+1}+\left(\frac{\sigma_{\Gamma_{\max }}\left\||\varepsilon|^{\gamma}\right\| \lambda_{\min }(K)+\Lambda}{\lambda_{\min }(K)}\right)\right\| \tilde{\theta} \|, \\
\leq-\sigma_{\Gamma_{\min }^{\gamma+1}}^{\gamma+\delta}(1-\delta)\|\tilde{\theta}\|^{\gamma+1}-\sigma_{\Gamma_{\min }^{\gamma+1}}^{\gamma+}\|\tilde{\theta}\| \gamma+1 \\
+\left(\frac{\sigma_{\Gamma_{\max }}\left\|\left.\varepsilon\right|^{\gamma}\right\| \lambda_{\min }(K)+\Lambda}{\lambda_{\min }(K)}\right)\|\tilde{\theta}\| .
\end{array}
$$

where $\delta \in(0,1)$. Since $\left\||\varepsilon|^{\gamma}\right\|=\sqrt{\sum_{i=1}^{m}\left|\varepsilon_{i}\right|^{2 \gamma}}=\|\varepsilon\|_{2 \gamma}^{\gamma}$, and by Hölder's inequality $\|\varepsilon\|_{2 \gamma} \leq m^{\frac{2-\gamma}{4 \gamma}}\|\varepsilon\|$ holds for all $2>2 \gamma>0$, it is given that

$$
\begin{aligned}
\dot{V}(t) \leq-\sigma_{\Gamma_{\min }}^{\gamma+1}(1-\delta) & \|\tilde{\theta}\|{ }^{\gamma+1}-\sigma_{\Gamma_{\min }^{\gamma+1}}^{\gamma+1}\|\tilde{\theta}\|^{\gamma+1} \\
& +\left(\frac{\sigma_{\Gamma_{\max }} m^{\frac{2-\gamma}{4}}\|\varepsilon\|^{\gamma} \lambda_{\min }(K)+\Lambda}{\lambda_{\min }(K)}\right)\|\tilde{\theta}\|,
\end{aligned}
$$

and then,

$$
\dot{V}(t) \leq-\sigma_{\Gamma_{\min }}^{\gamma+1}(1-\delta)\|\tilde{\theta}\|^{\gamma+1}, \forall\|\tilde{\theta}\| \geq \bar{\mu} .
$$

The rest of the proof follows the same steps as the proof of Corollary 1 providing that the solutions of system (5), with $\varepsilon \neq 0$, are globally ultimate bounded with its trajectories satisfying the bound given by (7).

Proof of Theorem 3: Consider the Lyapunov function (12) satisfying inequalities (13)-(14) with $c_{1}=2 \lambda_{\max }(K)$ and $c_{2}=2 \lambda_{\min }(K)$. Its time derivative along the trajectories of the error dynamics (9) is given by

$$
\begin{aligned}
\dot{V}(t) & =-\tilde{\theta}^{T} \Gamma\left(\left\lceil\Gamma^{T} \tilde{\theta}\right]^{\gamma_{1}}+\left\lceil\Gamma^{T} \tilde{\theta}\right]^{\gamma_{2}}\right)-\tilde{\theta}^{T} K^{-1} \Theta(\omega t), \\
& \leq-\left(\left\|\tilde{\theta}^{T} \Gamma\right\| \begin{array}{c}
\gamma_{1}+1 \\
\gamma_{1}+1
\end{array}+\left\|\tilde{\theta}^{T} \Gamma\right\| \begin{array}{l}
\gamma_{2}+1 \\
\gamma_{2}+1
\end{array}\right)+\frac{\Lambda}{\lambda_{\min }(K)}\|\tilde{\theta}\| .
\end{aligned}
$$

Then, since $\left\|\tilde{\theta}^{T} \Gamma\right\| \leq\left\|\tilde{\theta}^{T} \Gamma\right\|_{\gamma_{1}+1}$ and, by Hölder's inequality, $\left\|\tilde{\theta}^{T} \Gamma\right\| \leq n^{\frac{\gamma_{2}-1}{2\left(\gamma_{2}+1\right)}}\left\|\tilde{\theta}^{T} \Gamma\right\|_{\gamma_{2}+1}$ hold for all $\gamma_{2}+1>2>\gamma_{1}+1>$ $0, \dot{V}$ is bounded as follows

$$
\begin{aligned}
\dot{V}(t) \leq & -\left(\left\|\tilde{\theta}^{T} \Gamma\right\| \gamma_{1}+1+n^{\frac{1-\gamma_{2}}{2\left(\gamma_{2}+1\right)}}\left\|\tilde{\theta}^{T} \Gamma\right\| \gamma_{2}+1\right)+\frac{\Lambda}{\lambda_{\min }(K)}\|\tilde{\theta}\|, \\
\leq & -\sigma_{\Gamma_{\min } \gamma_{1}+1}^{\gamma_{2}} V^{\frac{\gamma_{1}+1}{2}} V^{\frac{\gamma_{1}+1}{2}}(t)-\frac{\sigma_{\Gamma_{\min }}^{\gamma_{2}+1} c_{2}^{\frac{\gamma_{2}+1}{2}}}{n^{\frac{\gamma_{2}-1}{2\left(\gamma_{2}+1\right)}}} V^{\frac{\gamma_{2}+1}{2}}(t) \\
& +\frac{2 \sqrt{c_{1}}}{c_{2}} \Lambda V^{\frac{1}{2}}(t) .
\end{aligned}
$$

Let us introduce the following inequalities

$$
\begin{aligned}
& V^{\gamma_{2}+1} \leq V^{\gamma_{1}+1} \leq V, \forall V \leq 1, \\
& V^{\gamma_{2}+1}>V^{\gamma_{1}+1}>V, \forall V>1 .
\end{aligned}
$$

Hence, from (14), (20) and the previous inequalities, when $V>1$ it is obtained that

$$
\begin{aligned}
\dot{V}(t) & \leq-\left(\frac{\sigma_{\Gamma_{\min }}^{\gamma_{2}+1}\left(2 \lambda_{\min }(K)\right)^{\frac{\gamma_{2}+1}{2}}}{n^{\frac{\gamma_{2}-1}{2\left(\gamma_{2}+1\right)}}}-\frac{\sqrt{2 \lambda_{\max }(K)}}{\lambda_{\min }(K)} \Lambda\right) V^{\frac{\gamma_{2}+1}{2}}(t), \\
& =-\alpha V^{\frac{\gamma_{2}+1}{2}}(t),
\end{aligned}
$$


that is negative definite for all $\lambda_{\min }(K) \quad>$ $\left(n^{\frac{\gamma_{2}-1}{2\left(\gamma_{2}+1\right)}} \sqrt{\lambda_{\max }(K)} \Lambda / 2^{\frac{\gamma_{2}}{2}} \sigma_{\Gamma_{\min }}^{\gamma_{2}+1}\right)^{2 /\left(\gamma_{2}+3\right)}=\bar{\lambda}_{1}$. Thus, for any $\tilde{\theta}(t)$ such that $V(\tilde{\theta}(0))>1$, the last inequality ensures $V(\tilde{\theta}(t)) \leq 1$ for $t \geq T_{1}=2 / \alpha\left(\gamma_{2}+1\right)$.

For the case when $V \leq 1$, it follows that

$$
\dot{V}(t) \leq-\sigma_{\Gamma_{\min }}^{\gamma_{1}+1}\left(2 \lambda_{\min }(K)\right)^{\frac{\gamma_{1}+1}{2}} V^{\frac{\gamma_{1}+1}{2}}(t)+\frac{\sqrt{2 \lambda_{\max }(K)}}{\lambda_{\min }(K)} \Lambda V^{\frac{1}{2}}(t) .
$$

Let us assume that $\gamma_{1}=0$. Hence, it follows that

$$
\begin{aligned}
\dot{V}(t) & \leq-\left(\sigma_{\Gamma_{\min }}\left(2 \lambda_{\min }(K)\right)^{\frac{1}{2}}-\frac{\sqrt{2 \lambda_{\max }(K)}}{\lambda_{\min }(K)} \Lambda\right) V^{\frac{1}{2}}(t), \\
& =-\beta V^{\frac{1}{2}}(t),
\end{aligned}
$$

which is negative definite for all $\lambda_{\min }(K)$ $\left(\sqrt{\lambda_{\max }(K)} \Lambda / \sigma_{\Gamma_{\min }}\right)^{2 / 3}=\bar{\lambda}_{2}$. Then, for any $\tilde{\theta}(t)$ such that $V(\tilde{\theta}(T)) \leq 1$, it is derived that $V(\tilde{\theta}(t))=0$ for $t \geq T_{1}+(2 / \beta)$. Therefore, $V(\tilde{\theta}(t))=0$, for all $t \geq 2\left(\alpha\left(\gamma_{2}+1\right)+\beta\right) / \alpha \beta\left(\gamma_{2}+1\right)$, and all $\tilde{\theta}(t)$

Thus, based on Lemma 1 in [16], the system (9) is GFxTS.

Proof of Corollary 3: This proof follows the same steps as the previous proof except for the case when $V \leq 1$. Let us consider (20), i.e.

$$
\dot{V}(t) \leq-\sigma_{\Gamma_{\min }}^{\gamma_{1}+1}\|\tilde{\theta}\| \gamma_{1}+1-\frac{\sigma_{\Gamma_{\min }}^{\gamma_{2}+1}}{n^{\frac{\gamma_{2}-1}{2\left(\gamma_{2}+1\right)}}}\|\tilde{\theta}\| \gamma_{2}+1+\frac{\Lambda}{\lambda_{\min }(K)}\|\tilde{\theta}\| .
$$

Taking into account that inequalities (21) and (22) hold for every $\gamma_{2}+1>\gamma_{1}+1>1$, then when $V \leq 1$, it follows that

$$
\begin{aligned}
\dot{V}(t) & \leq-\sigma_{\Gamma_{\min }}^{\gamma_{1}+1}\|\tilde{\theta}\| \gamma^{\gamma_{1}+1}+\frac{\Lambda}{\lambda_{\min }(K)}\|\tilde{\theta}\|, \\
& \leq-\sigma_{\Gamma_{\min }}^{\gamma_{1}+1}(1-\delta)\|\tilde{\theta}\|^{\gamma_{1}+1}-\sigma_{\Gamma_{\min }}^{\gamma_{1}+1} \delta\|\tilde{\theta}\| \gamma^{\gamma_{1}+1}+\frac{\Lambda}{\lambda_{\min }(K)}\|\tilde{\theta}\|,
\end{aligned}
$$

where $\delta \in(0,1)$. Hence,

$$
\dot{V}(t) \leq-\sigma_{\Gamma_{\min }}^{\gamma_{1}+1}(1-\delta)\|\tilde{\theta}\|^{\gamma_{1}+1}, \forall c_{1}^{\frac{1}{2}} \geq\|\tilde{\theta}\| \geq \mu_{f} .
$$

From (24), it follows that

$$
\dot{V}(t) \leq-\sigma_{\Gamma_{\min }}^{\gamma_{1}+1}(1-\delta) c_{2}^{\frac{\gamma_{1}+1}{2}} V^{\frac{\gamma_{1}+1}{2}}(t)
$$

and by the comparison principle one gets

$$
V(t) \leq\left(V^{\frac{1-\gamma_{1}}{2}}(0)-\frac{\sigma_{\Gamma_{\min }}^{\gamma_{1}+1}(1-\delta) c_{2}^{\frac{\gamma_{1}+1}{2}}\left(1-\gamma_{1}\right)}{2} t\right)^{\frac{2}{1-\gamma_{1}}},
$$

then, the last inequality ensures that $\tilde{\theta}$ satisfies the following bound

$$
\begin{aligned}
& \|\tilde{\theta}(t)\| \leq \sqrt{c_{1}} \times \\
& \left(c_{2}^{\frac{\gamma_{1}-1}{2}}\|\tilde{\theta}(0)\|{ }^{1-\gamma_{1}}-\frac{\sigma_{\Gamma_{\min }}^{\gamma_{1}+1}(1-\delta) c_{2}^{\frac{\gamma_{1}+1}{2}}\left(1-\gamma_{1}\right)}{2} t\right)^{\frac{1}{1-\gamma_{1}}},
\end{aligned}
$$

for all $t<T(\tilde{\theta}(0))$; while for all $t \geq T(\tilde{\theta}(0))$, from (13), it is obtained that $\tilde{\theta}$ is bounded as in (10), i.e. $\|\tilde{\theta}\| \leq$ $\sqrt{\lambda_{\max }(K) / \lambda_{\min }(K)} \min \left(c_{1}^{1 / 2}, \mu_{f}\right)$.

Therefore, it is concluded that the solutions of system (9) are globally ultimate bounded with its trajectories satisfying the bound given by (10).

Proof of Corollary 4: Assume, in the component-wise sense, that $\left|\left(\Gamma^{T} \tilde{\theta}\right)_{i}\right| \geq\left|\varepsilon_{i}\right|$, for all $i=\overline{1, n}$. Then, it implies that $\operatorname{sign}\left(\Gamma^{T} \tilde{\theta}-\right.$ $\varepsilon)=\operatorname{sign}\left(\Gamma^{T} \tilde{\theta}\right)$. Consider that for any $x, \bar{x} \in \mathbb{R}$ and $\gamma_{2}>1$, the inequality $|x+\bar{x}|^{\gamma_{2}} \leq 2^{\gamma_{2}-1}\left(|x|^{\gamma_{2}}+|\bar{x}|^{\gamma_{2}}\right)$ holds [25]. Thus, defining $x=\left(\Gamma^{T} \tilde{\theta}\right)_{i}-\varepsilon_{i}$ and $\bar{x}=\varepsilon_{i}$, it follows that

$$
\begin{gathered}
\left|\left(\Gamma^{T} \tilde{\theta}\right)_{i}\right|^{\gamma_{2}}=\left|\left(\Gamma^{T} \tilde{\theta}\right)_{i}-\varepsilon_{i}+\varepsilon_{i}\right|^{\gamma_{2}}, \\
\leq 2^{\gamma_{2}-1}\left(\left|\left(\Gamma^{T} \tilde{\theta}\right)_{i}-\varepsilon_{i}\right|^{\gamma_{2}}+\left|\varepsilon_{i}\right|^{\gamma_{2}}\right), \\
\Rightarrow\left|\left(\Gamma^{T} \tilde{\theta}\right)_{i}\right|^{\gamma_{2}-} 2^{\gamma_{2}-1}\left|\varepsilon_{i}\right|^{\gamma_{2}} \leq 2^{\gamma_{2}-1}\left|\left(\Gamma^{T} \tilde{\theta}\right)_{i}-\varepsilon_{i}\right|^{\gamma_{2}},
\end{gathered}
$$

for all $i=\overline{1, n}$, and then component-wisely

$$
-\left|\Gamma^{T} \tilde{\theta}-\varepsilon\right|^{\gamma_{2}} \leq-2^{1-\gamma_{2}}\left|\Gamma^{T} \tilde{\theta}\right|^{\gamma_{2}}+|\varepsilon|^{\gamma_{2}},
$$

holds for all $\gamma_{2}>1$. Taking into account the previous inequality, this proof follows the same steps as the proof of Theorem 3, Corollary 2 and Corollary 3 .

\section{REFERENCES}

[1] H. Huijberts, H. Nijmeijer, and R. Willems, "System identification in communication with chaotic systems," IEEE Transactions on Circuits and Systems - I: Fundamental Theory and Applications, vol. 47, no. 6, pp. 800-808, 2000.

[2] Q. Zhongfang, "Distributed parameter system identification and its application to geophysical parameter identification," in Proceedings of the IEEE International Conference on Industrial Technology, (Guangzhou), pp. 799-802, 1994.

[3] W. Sun, H. Li, and Q. Han, "Idenitification of mechanical parameters of hard-coating materials with strain-dependence," Journal of Mechanical Science and Technology, vol. 28, no. 1, pp. 81-92, 2014.

[4] J. Hasenauer, S. Waldherr, K. Wagner, and F. Allgöwer, "Parameter identification, experimental design and model falsification for biological network models using semidefinite programming," IET Systems Biology, vol. 4 , no. 2, pp. 119-130, 2010.

[5] L. Ljung, System Identification: Theory for the User. New Jersey: Prentice Hall Information and System Sciences Series, second ed., 1999.

[6] R. Isermann and M. Münchhof, Identification of Dynamic Systems, An Introduction with Applications. Berlin: Springer-Verlag, 1st ed., 2011.

[7] J. Chen, G. Zhang, and Z. Li, "On-line parameter estimation for a class of time-varying continuous systems with bounded disturbances," International Journal of Adaptive Control and Signal Processing, no. 25, pp. 18-32, 2011.

[8] J. Li, Y. Zheng, and Z. Lin, "Recursive identification of time-varying systems: Self-tuning and matrix rls algorithms," Systems \& Control Letters, vol. 66, pp. 104-110, 2014.

[9] Y. Zhu and P. Pagilla, "Adaptive estimation of time-varying parameters in linearly parametrized systems," Journal of Dynamic Systems, Measurement, and Control, vol. 128, pp. 691-695, 2005.

[10] A. Polyakov and L. Fridman, "Stability notions and lyapunov functions for sliding mode control systems," Journal of the Franklin Institute, vol. 351, no. 4, pp. 1831-1865, 2014.

[11] J. A. Moreno and E. Guzman, "A new recursive finite-time convergent parameter estimation algorithm," in In Proccedings of the 18th IFAC World Congress, vol. 18, (Milano, Italy), pp. 3439-3444, 2011.

[12] J. Moreno and M. Osorio, "Strict lyapunov functions for the supertwisting algorithm," IEEE Transactions on Automatic Control, vol. 57, no. 4, pp. 1035-1040, 2012.

[13] J. Davila, L. Fridman, and A. Poznyak, "Observation and identification of mechanical systems via second order sliding modes," International Journal of Control, vol. 79, no. 10, pp. 1251-1262, 2006.

[14] J.-X. Xu, Y.-J. Pan, and T.-H. Lee, "A VSS identification scheme for time-varying parameters," Automatica, vol. 39, pp. 727-734, 2003.

[15] V. Adetola and M. Guay, "Finite-time parameter estimation in adaptive control of nonlinear systems," IEEE Transactions on Automatic Control, vol. 53, no. 3, pp. 807-811, 2008.

[16] A. Polyakov, "Nonlinear feedback design for fixed-time stabilization of linear control systems," Automatic Control, IEEE Transactions on, vol. 57, no. 8, pp. 2106-2110, 2012.

[17] H. Ríos, D. Efimov, A. Polyakov, and W. Perruquetti, "Homogeneous time-varying systems: Robustness analysis," IEEE Transactions on Automatic Control, 2016. DOI:10.1109/TAC.2016.2540806.

[18] H. Ríos, D. Efimov, L. Fridman, J. Moreno, and W. Perruquetti, "Homogeneity based uniform stability analysis for time-varying systems," IEEE Transactions on Automatic Control, vol. 61, no. 3, pp. 725-734, 2016.

[19] H. Khalil, Nonlinear Systems. New Jersey, U.S.A.: Prentice Hall, 2002.

[20] P. Dorato, Short-time stability in linear time-varying systems. $\mathrm{PhD}$ thesis, Polytechnic Institute of Brooklyn, New York, 1961.

[21] L. Weiss and E. Infante, "On the stability of systems defined over a finite time interval," Proc. of the National Academy of Sciences, vol. 54, pp. 440-448, 1965.

[22] E. Roxin, "On finite stability in control systems," Rendiconti del Circolo Matematico di Palermo, vol. 15, pp. 273-283, 1966.

[23] S. Bhat and D. Bernstein, "Geometric homogeneity with applications to finite-time stability," Mathematics of Control, Signals and Systems, vol. 17, pp. 101-127, 2005.

[24] J. Rueda-Escobedo and J. Moreno, "Discontinuous gradient algorithm for finite-time estimation of time-varying parameters," International Journal of Control, vol. 89, no. 9, 2016.

[25] D. Mitrinović, Analytic Inequalities, vol. 165 of Grundlehren der mathematischen Wissenschaften. Springer-Verlang Berlin Heidelberg, 1970. 